\title{
Application of FLiP Method for Differentiation of Mycobacterium tuberculosis Strains in Comparison to Commonly Used Methods, Spoligotyping and MIRU-VNTR Typing
}

\author{
ANNA ŻACZEK ${ }^{1}$, ANNA BRZOSTEK ${ }^{2}$, ALINA GÓRNA ${ }^{2,3}$, ANNA SAJDUDA ${ }^{3}$, ARKADIUSZ WOJTASIK² \\ and JAROSŁAW DZIADEK ${ }^{1,2 *}$ \\ ${ }^{1}$ Department of Biochemistry and Cell Biology, University of Rzeszów, Poland \\ ${ }^{2}$ Institute of Medical Biology, Polish Academy of Sciences, Łódź, Poland \\ ${ }^{3}$ Department of Genetics of Microorganisms, University of Łódź, Poland
}

Received 11 April 2012, revised 27 September 2012, accepted 28 September 2012

\section{Abstract}

The current "gold standard" in molecular epidemiological studies of Mycobacterium tuberculosis is IS6110 RFLP based on IS6110 polymorphism. However PCR-based methods are becoming increasingly important. Recently, fast ligation-mediated PCR (FLiP), based on IS6110 polymorphism was proposed. In this study, the discriminatory power of FLiP, spoligotyping and MIRU-VNTR typing, in differentiation of M. tuberculosis isolates was compared. The discriminatory index (HGI) of spoligotyping, MIRU-VNTR analysis, and FLiP was 0.653 , 0.837, and 0.917 , respectively. This indicates that FLiP allows a high level of differentiation among M. tuberculosis strains and it might be a valuable alternative to the other typing methods.

Ke y w o rds: Mycobacterium tuberculosis, FLiP, MIRU-VNTR typing, spoligotyping

Tuberculosis (TB) is an infectious disease caused by Mycobacterium tuberculosis (M.tb.), one of the most dangerous human pathogens. Recently numerous molecular techniques have been developed to support and reinforce conventional epidemiology of TB. Molecular methods provide the rates of active transmission and the understanding of transmission dynamics, helping additionally to discover cross-contamination (van Soolingen et al., 2003). The differentiation ability of each method depends on the type and level of polymorphism detected. $M$. $t b$. has relatively low genetic variability due to its specific life cycle, involving long doubling time and periods of latency (Gutacker et al., 2002). The diversity of mycobacterial genomes is associated with rearrangements within the chromosome resulting from recombination, mutations and transposition of mobile genetic elements (Eisenach et al., 1988).

IS6110 is an insertion sequence frequently used in genetic analyses of M. tb. (Thierry et al., 1990). IS6110 RFLP (restriction fragment length polymorphism) is a molecular method based on differences in copy number and variability in the chromosomal locations of IS6110. Due to high standardization and wide use it is considered a reference method (van Embden et al., 1993). However, this method is laborious, expensive and requires a large amount of chromosomal DNA. Therefore, several alternative PCR-based techniques have been developed.

Spoligotyping requires less DNA than the standard IS6110 RFLP (Kamerbeek et al., 1997). This method is based on polymorphism in the chromosomal direct repeat (DR) locus. It consists of PCR amplification of DRs and subsequent hybridization of the products with 43 synthetic oligonucleotides immobilized on a membrane. Spoligotyping is often used as a quick screening method, especially for differentiation of strains with similar IS6110 RFLP patterns or strains with low number (less than 6) of IS6110 copies (Mathema et al., 2006).

Another currently used PCR-based method for genotyping $M$. $t b$. strains is MIRU-VNTR (mycobacterial interspersed repetitive units-variable number of tandem repeats) typing. This method is based on variable number of tandem repeats. The 12, 15 or 24 loci, selected from 61 MIRU-VNTR sequences, present in the $M$. $t b$. genome, allow the differentiation of isolates (Supply et al., 2006). MIRU-VNTR typing based on 12 or 15 loci has a lower discriminatory power than IS6110 RFLP, but this can be increased by combining it with spoligotyping (Kremer et al., 1999a, b; Blackwood et al., 2004; Covan etal., 2005; Mathema et al., 2006;

\footnotetext{
* Corresponding author: J. Dziadek, Institute of Medical Biology, Polish Academy of Sciences, ul. Lodowa 106, 93-232 Łódź, Poland;
} phone: +48 4227236 10; fax: +48 4227236 30; e-mail: jdziadek@toya.net.pl; jdziadek@cbm.pan.pl 


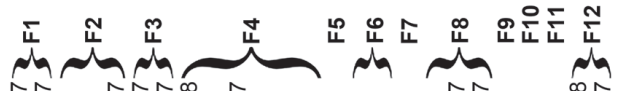

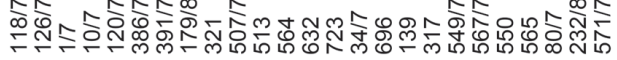
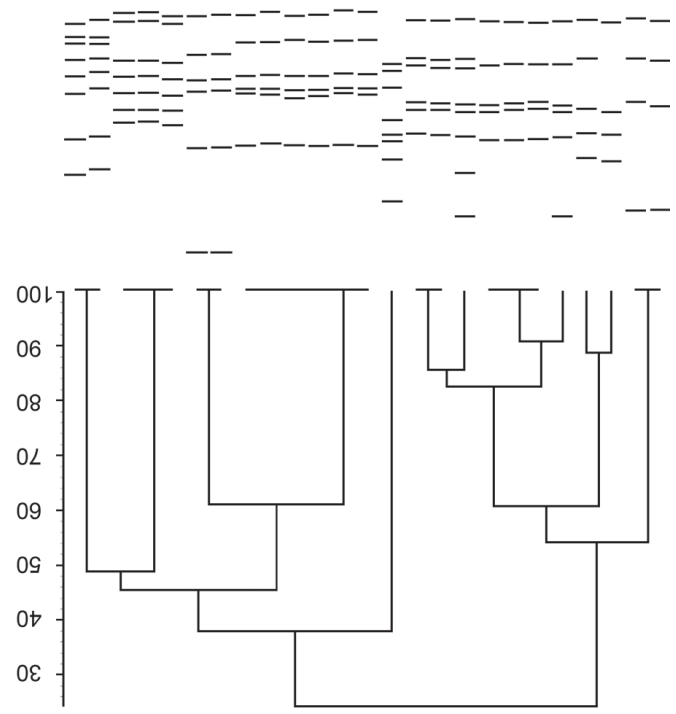

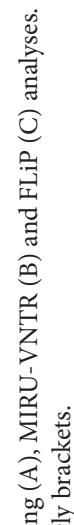

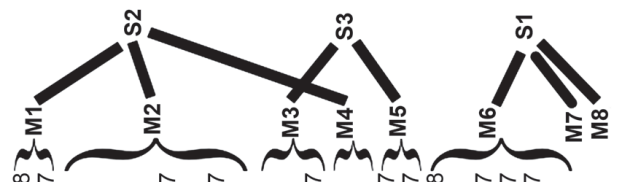

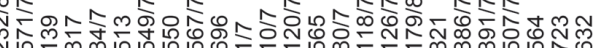
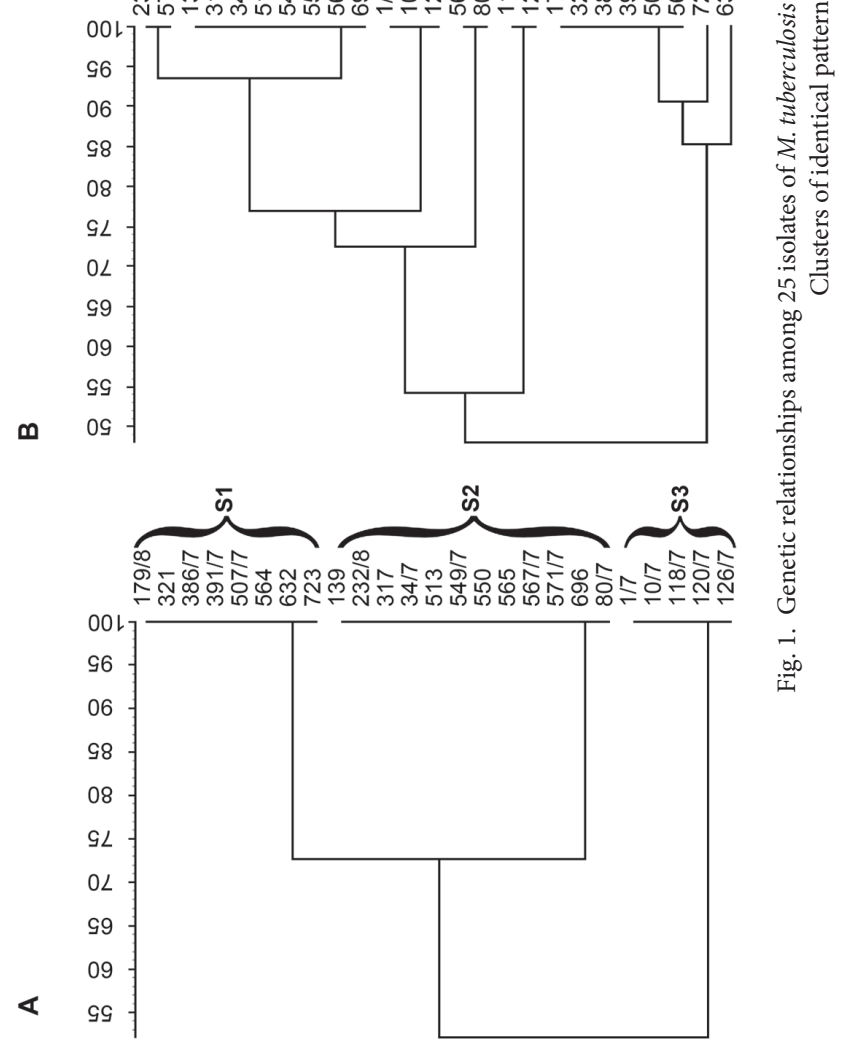
Sajduda et al., 2006; Allix-Béguec et al., 2008). Further extension of the set of loci to 24 sequences resulted in higher resolution power comparable to that of the reference method (Maes et al., 2008).

Fast ligation-mediated PCR (FLiP) is a novel method, based on IS6110 polymorphism. It involves digestion of chromosomal DNA by restriction enzyme HhaI and ligation with a synthetic oligonucleotide adaptor sequence (Reisig et al., 2005). Such product is then PCR amplified using primers complementary to the IS6110 and adaptor sequence, and resolved by polyacrylamide gel electrophoresis. The discriminatory power of FLiP was reported to be close to that of the IS6110 RFLP (Kremer et al., 2005; Reisig et al., 2005). However, very few analyses of $M$. $t b$. strains were performed using FLiP, and its comparison to other commonly used PCR-based methods is therefore required.

In this study, we used FLiP to estimate molecular relationships among 25 strains of $M$. $t b$., isolated from TB patients in 2006-2007. FLiP analysis was performed as originally described by Reisig et al., 2005. FLiP genotyping data were compared to the results previously obtained by 15-locus MIRU-VNTR typing and spoligotyping, two PCR-based methods commonly used in molecular epidemiology of TB (Krawczyk et al., 2011).

Spoligotyping grouped the analyzed strains into three types: S1, S2, and S3, consisting of 8, 12 and 5 strains, respectively (Fig. 1A.). The discriminatory power of spoligotyping, expressed as HGI (HunterGaston index; Hunter and Gaston, 1988), was 0.653. The 15-locus MIRU-VNTR analysis divided the 25 strains into 8 classes: M1-M8 (Fig. 1B.). Within each of the types S1 and S2, the 15-locus MIRU-VNTR distinguished three classes: M6, M7, M8, and M1, M2, M4, respectively. Type S3 was subdivided into classes M3 and M5. The classes M2, M6 and M3 contained 8, 6 and 3 strains, respectively. The classes M1, M4, and M5 contained two strains each, whereas classes M7 and M8 were represented by single strains. The HGI for 15-locus MIRU-VNTR typing was 0.837 . Combining it with spoligotyping increased HGI to 0.930 (data not shown).

The FLiP method subdivided 25 strains tested into 12 clusters, leading to further differentiation of strains belonging to classes M4 and M6 (Fig. 1C.). Class M4 was subdivided into single strains F10 and F11, and class M6 was split up into clusters F3 and F4. Class M2 was diversified into four clusters: F6, F7, F8, and F9 with the exception of strain no. 513. This strain was included into the cluster F4 together with strain no. 632, belonging to the class M8 in MIRU-VNTR typing. In comparison with the two other methods, FLiP showed the highest discriminatory potential $(\mathrm{HGI}=0.917)$ in differentiation of the strains tested.

In conclusion, spoligotyping can be used as a screening method for molecular epidemiological investiga- tions of large numbers of $M . t b$. strains. The high discriminatory power presented by FLiP suggests it as an interesting alternative method for the widely used MIRU-VNTR typing. However, in large numbers of strains tested, the use of MIRU-VNTR typing could be more appropriate, since it requires determination of the size of a single PCR product instead of comparison of patterns, as in FLiP. FLiP appears a relatively simple and fast method, reliably discriminating $M$. $t b$. strains. However, further studies of strains from worldwide distribution would be necessary to prove its wide applicability.

\section{Acknowledgments}

This work was supported by grant no. N N302 111338 from the National Science Centre, and partially performed within the project (POPW.01.03.00-18-018/09) "Center of Applied Biotechnology and Basic Sciences", supported by the Operational Program "Development of Eastern Poland 2007-2013".

\section{Literature}

Allix-Béguec C., D. Harmsen, T. Weniger, P. Supply and S. Niemann. 2008. Evaluation and strategy for use of MIRU-VNTRplus, a multifunctional database for online analysis of genotyping data and phylogenetic identification of Mycobacterium tuberculosis complex isolates. J. Clin. Microbial. 46: 2692-2699.

Blackwood K.S., J.N. Wolfe and A.M. Kabani. 2004. Application of mycobacterial interspersed repetitive unit typing to Manitoba tuberculosis cases: can restriction fragment length polymorphism be forgotten? J. Clin. Microbiol. 42: 5001-5006.

Covan L.S., L. Diem, T. Monson, P. Wand, D. Temporado, T.V. Oemig and J.T. Crawford. 2005. Evaluation of a two-step approach for large-scale, prospective genotyping of Mycobacterium tuberculosis isolates in the United States. J. Clin. Microbiol. 43: 688-695.

Eisenach K.D., J.T. Crawford and J.H. Bates. 1988. Repetitive DNA sequences as probes for Mycobacterium tuberculosis. J. Clin. Microbiol. 26: 2240- 2245 .

Gutacker M.M., J.C. Smoot, C.A.L. Migliaccio, S.M. Ricklefs, S. Hua, D.V. Cousins, E.A. Graviss, E. Shashkina, B.N. Kreiswirth and J.M. Musser. 2002. Genome-wide analysis of synonymous single nucleotide polymorphisms in Mycobacterium tuberculosis complex organisms: resolution of genetic relationships among closely related microbial strains. Genetics 162: 1533-1543.

Hunter P.R. and M.A. Gaston. 1988. Numerical index of the discriminatory ability of typing systems: an application of Simpson's index of diversity. J. Clin. Microbiol. 26: 2465-2466.

Kamerbeek J., L. Schouls, A. Kolk, M. van Agterveld, D. van Soolingen, S. Kuijper, A. Bunschoten, H. Molhuizen, R. Shaw, M. Goyal and others. 1997. Simultaneous detection and strain differentiation of Mycobacterium tuberculosis for diagnosis and epidemiology. J. Clin. Microbiol. 35: 907-914.

Krawczyk M., A. Brzostek, A. Gorna, K. Knapska, M. Ziolkiewicz, A. Wojtasik and J. Dziadek. 2011. Epidemiological analysis of Mycobacterium tuberculosis strains isolated in Lodz, Poland. Int. J. Tuberc. Lung Dis. 15: 1252-1258.

Kremer K., C. Arnold, A. Cataldi, M.C. Gutiérrez, W.H. Haas, S. Panaiotov, R.A. Skuce, P. Supply, A.G.M. van der Zanden and D. van Soolingen. 1999a. Discriminatory power and reproducibility of novel DNA typing methods for Mycobacterium tuberculosis complex strains. J. Clin. Microbiol. 43: 5628-5638. 
Kremer K., D. van Soolingen, R. Frothingham, W.H. Haas, P.W. Hermans, C. Martín, P. Palittapongarnpim, B.B. Plikaytis, L.W. Riley, M.A. Yakrus, J.M. Musser and J.D. van Embden. 1999b. Comparison of methods based on different molecular epidemiological markers for typing of Mycobacterium tuberculosis complex strains: interlaboratory study of discriminatory power and reproducibility. J. Clin. Microbiol. 37: 2607-2618.

Maes M., K. Kremer, D. van Soolingen, H. Takiff and J.H. de Waard. 2008. 24-locus MIRU-VNTR genotyping is a useful tool to study the molecular epidemiology of tuberculosis among Warao Amerindians in Venezuela. Tuberculosis 5: 490-494.

Mathema B., N.E. Kurepina, P.J. Bifani and B.N. Kreiswirth. 2006. Molecular epidemiology of tuberculosis: current insights. Clin. Microbiol. Rev. 19: 658-685.

Reisig, F., K. Kremer, B. Amthor, D. van Soolingen and W.H. Haas. 2005. Fast ligation-mediated PCR, a fast and reliable method for IS6110-based typing of Mycobacterium tuberculosis complex. J. Clin. Microbiol. 43: 5622-5627.

Sajduda A., J. Dziadek, R. Kotłowski and F. Portaels. 2006. Evaluation of multiple genetic markers for typing drug-resistant $\mathrm{Myco}$ - bacterium tuberculosis strains from Poland. Diagn. Microbiol. Infect. Dis. 55: 59-64.

Supply P., C. Allix, S. Lejean, M. Cardosso-Oelemann, S. RushGerdes, E. Willery, E. Savine, P. de Haas, H. van Deutekom, S. Roring and others. 2006. Proposal for standardization of optimized mycobacterial interspersed repetitive unit-variable-number tandem repeat typing of Mycobacterium tuberculosis. J. Clin. Microbiol. 44: 4498-4510.

Thierry D., M.D. Cave, K.D. Eisenach J. T Crawford, J.H. Bates, B. Gicquel and J. L. Guesdon. 1990. IS6110, an IS-like element of Mycobacterium tuberculosis complex. Nucleic Acids Res. 18: 188-193.

van Embden J.D., M.D. Cave, J.T. Crawford, J.W. Dale, K.D. Eisenach, B. Gicquel, P. Hermans, C. Martin, R. McAdam and T. Shinnick. 1993. Strain identification of Mycobacterium tuberculosis by DNA fingerprinting: recommendations for a standardized methodology. J. Clin. Microbiol. 31: 406-409.

Van Soolingen D., K. Kremer and E. Vynnycky. 2003. New perspectives in the molecular epidemiology of tuberculosis. Mycobacteria and TB. 2: 17-45. 